\title{
Complement Component C3 Binds to the A3 Domain of von Willebrand Factor
}

\author{
Jennifer G. Nolasco ${ }^{1}$ Leticia H. Nolasco ${ }^{2}$ \\ Joel L. Moake ${ }^{2}$ Miguel A. Cruz ${ }^{1,3}$ \\ ${ }^{1}$ Section of Cardiovascular Research, Department of Medicine, Baylor \\ College of Medicine, Houston, Texas, United States \\ ${ }^{2}$ Department of Bioengineering, Rice University, Houston, Texas, \\ United States \\ ${ }^{3}$ Center for Translational Research on Inflammatory Diseases (CTRID), \\ Michael E. DeBakey VA Medical Center, Houston, Texas, United States \\ ${ }^{4}$ Department of Molecular Medicine, MERU-Roon Research Center on \\ Vascular Biology, The Scripps Research Institute, La Jolla, California, \\ United States
}

TH Open 2018;2:e338-e345.
Address for correspondence Miguel A. Cruz, PhD, Cardiovascular Research Section, Baylor College of Medicine, 2002 Holcombe Blvd., Bldg. 109, Houston, TX 77030, United States (e-mail: miguelc@bcm.edu).

\author{
Abstract \\ Keywords \\ - complement \\ component $\mathrm{C} 3$ \\ - von Willebrand factor \\ - alternative \\ complement pathway \\ - thrombosis \\ - inflammation
}

von Willebrand factor (VWF) is a multimeric protein composed of monomeric subunits ( $\sim 280 \mathrm{kD}$ ) linked by disulfide bonds. During hemostasis and thrombosis, ultralarge (UL) VWF (ULVWF) multimers initiate platelet adhesion. In vitro, human C3 binds to ULVWF multimeric strings secreted by and anchored to human endothelial cell to promote the assembly and activation of $\mathrm{C} 3$ convertase ( $\mathrm{C} 3 \mathrm{bBb}$ ) and $\mathrm{C} 5$ convertase ( $\mathrm{C} 3 \mathrm{bBbC} 3 \mathrm{~b}$ ) of the alternative complement pathway (AP). The purified and soluble $\mathrm{C} 3$ avidly binds to recombinant human VWF A1A2A3, as well as the recombinant isolated human VWF A3 domain. Notably, the binding of soluble human ULVWF multimers to purified human C3 was blocked by addition of a monovalent Fab fragment antibody to the VWFA3 domain. We conclude that the A3 domain in VWF/ULVWF contains a docking site for C3. In contrast, purified human $\mathrm{C} 4$, an essential component of the classical and lectin complement pathways, binds to soluble, isolated A1, but not to ULVWF strings secreted by and anchored to endothelial cells. Our findings should facilitate the design of new therapeutic agents to suppress the initiation of the AP on ULVWF multimeric strings during thrombotic and inflammatory disorders.

\section{Introduction}

von Willebrand factor (VWF) is a multimeric protein composed of monomeric subunits $(\sim 280 \mathrm{kD})$ linked into large polymers by disulfide bonds. VWF multimers are synthesized in endothelial cells (ECs) and megakaryocytes, where the multimers are stored in Weibel-Palade bodies (ECs) or $\alpha$ granules (megakaryocytes and platelets). ${ }^{1}$ During hemostasis and thrombosis, stimulated human vascular ECs secrete and anchor ultralarge (UL)VWF (ULVWF) multimers in hyperadhesive long string-like structures that initiate platelet adhesion., ${ }^{2,3}$
Recently, we showed that EC-secreted/anchored ULVWF string-like structures, in addition to initiating platelet adhesion, serve also as surfaces for assembling the components of the alternative complement pathway (AP). ${ }^{4}$ In vitro, both hydrated $\mathrm{C} 3\left[\mathrm{C} 3\left(\mathrm{H}_{2} \mathrm{O}\right)\right]$ (designated in this paper as " $\mathrm{C} 3$ ") and $\mathrm{C} 3 \mathrm{~b}$ ( $\mathrm{C} 3$ after cleavage of the small peptide, $\mathrm{C} 3 \mathrm{a}$ ) are active forms of $\mathrm{C} 3$ that share domains enabling them to attach to surfaces and initiate activation of the $\mathrm{AP}^{5,6}$ Both hydrated $\mathrm{C} 3$ (C3) and C3b bind to ECanchored ULVWF multimeric strings and initiate assembly on the strings of $\mathrm{C} 3$ convertase $(\mathrm{C} 3 \mathrm{bBb})$ and $\mathrm{C} 5$ convertase (C3bBbC3b). ${ }^{4}$ This may be an important molecular mechanism received

May 23, 2018

accepted after revision

August 16, 2018
DOI https://doi.org/

10.1055/s-0038-1672189. ISSN 2512-9465. (c) 2018 Georg Thieme Verlag KG
Stuttgart · New York

License terms

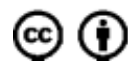


for initiation and activation of the AP. In contrast, there is little or no binding of C4 to EC-anchored ULVWF strings, ${ }^{4}$ indicating that the classical and lectin complement pathways, which require C4, are not also activated under these conditions.

To identify the precise location for $\mathrm{C} 3$ binding to $\mathrm{VWF}$, we investigated the interaction of $\mathrm{C} 3, \mathrm{C} 3 \mathrm{~b}$, and $\mathrm{C} 4$ with VWF A1, $A 2$, and A3 domains either linked together as VWF A1A2A3 or as individual, isolated recombinant $\mathrm{A} 1, \mathrm{~A} 2$, or $\mathrm{A} 3$ domains. The recombinant VWF A1A2A3 protein is referred to in figures as “TD." We identified VWFA3 domain as containing the docking site for C3. The results reported here will help clarify the molecular mechanisms underlying the cross-talk between coagulation and complement activation relevant in host defense. This will help design new therapeutic agents to suppress activation of the AP on ULVWF strings during thrombotic and inflammatory disorders.

\section{Materials and Methods}

\section{Antibodies and Proteins}

We used monoclonal anti-His antibody (GenScript, New Jersey, United States), goat anti-VWF antibody (A80-138, Bethyl Labs, Texas, United States), rabbit anti-VWF antibody (Ramco Laboratories, Texas, United States), horseradish peroxidase (HRP) conjugated goat anti-rabbit immunoglobulin G (IgG) and rabbit anti-goat IgG (Pierce Antibodies, Illinois, United States), and a CM series sensor chip (GE Health Care, New Jersey, United States), Antibody MR-5 is a mouse monoclonal IgG1 that binds to a specific sequence (residues 1711-1761) in the A3 domain of human VWF. ${ }^{7}$ It was produced at The Scripps Research Institute, where heavy and light chain of the $\operatorname{IgG}$ were cloned from hybridoma cDNA and engineered for expression as monovalent Fab in Drosophila melanogaster S2 cells. Highly purified C3, C3b, and C4, as well as monospecific anti-C3 and anti-C4 antibodies, were purchased from Complement Technologies (TX). Recombinant VWF A1A2A3 with His tag was expressed (and glycosylated) in mammalian cells (HEK293T) and purified as previously described. ${ }^{8}$ Recombinant individual VWF A1, A2, and A3 domains with His tag were expressed in Escherichia coli (in nonglycosylated forms) and purified as described. ${ }^{9} \mathrm{VWF}$ multimers enriched in ULVWF forms were obtained as we previously described ${ }^{10,11}$ from histamine-stimulated cultured human umbilical vein EC supernatant, and concentration was determined using a VWF enzyme-linked immunosorbent assay (ELISA) kit (Aviva System Biology, California, United States).

\section{ELISA Binding Assay}

Recombinant human VWF A1A2A3 or recombinant human individual $\mathrm{A} 1, \mathrm{~A} 2$, or $\mathrm{A} 3$ domains were incubated overnight with purified human $\mathrm{C} 3, \mathrm{C} 3 \mathrm{~b}$, or $\mathrm{C} 4$ at $4^{\circ} \mathrm{C}$ to allow complex formation. The complexes were captured with anti-His antibody and detected by anti-C3 or anti-C4, HRP-conjugated secondary anti-IgG antibody, and the chromogenic substrate, 3,3',5,5'tetramethylbenzidine (TMB, Life technologies, Massachusetts, United States) was used for detection and quantification. The color reaction was read at $450 \mathrm{nM}$ in a spectrophotometer.
Specifically, $100 \mu \mathrm{L}$ of the mixtures of VWF domains and $\mathrm{C} 3, \mathrm{C} 3 \mathrm{~b}$, or $\mathrm{C} 4$ were pipetted in duplicate and then incubated in High Bind Stripwells (Greiner Bio-One, Germany) with immobilized mouse monoclonal anti-His tag antibody (160 $\mathrm{ng} / \mathrm{mL}$ ) (Genscript, NJ) for 2 hours at $37^{\circ} \mathrm{C}$. Either VWF A1A2A3 or any of the individual A domains with bound C3, $\mathrm{C} 3 \mathrm{~b}$, or C4 was captured by the anti-His antibody immobilized onto the wells. Bound C3, C3b, or C4 (10 $\mu \mathrm{g} / \mathrm{mL})$ to 100 $\mu \mathrm{g} / \mathrm{mL}$ of either the VWF A1A2A3 or individual A1, A2, or A3 domain was detected by either goat anti-human $C 3$ or antiC4 plus rabbit anti-goat antibody linked to HRP (Pierce Antibody, Illinois, United States). Detection and quantification in the spectrophotometer was with TMB, as above. Bovine serum albumin in phosphate-buffered saline (PBS; $1 \%$ ) was used as negative control.

\section{Binding Kinetics of Complement Proteins and Recombinant VWF Domains}

Surface plasmon resonance (SPR) binding studies were performed using a BIAcore 3000 system (BIAcore, Piscataway, New Jersey, United States), as previously described. ${ }^{12,13}$ Either $50 \mu \mathrm{g} / \mathrm{mL}$ of C3, C4, or a VWF A domain protein in $50-\mathrm{mM}$ sodium acetate ( $\mathrm{pH}$ 5.0) was covalently coupled via amine coupling to a sensor chip (CM5) as directed by the manufacturer. The binding assays were performed in $10-\mathrm{mM}$ HEPES, $150-\mathrm{mM} \mathrm{NaCl}, 0.005 \%$ Tween-20, pH 7.4 at $25^{\circ} \mathrm{C}$ at a flow rate of $10 \mu \mathrm{L} / \mathrm{min}$. An activated blank channel was used as control for nonspecific binding correction. Binding at equilibrium was determined at a series of concentrations of the perfused protein ( $\mathrm{C} 3, \mathrm{C} 4$, or a VWF A domain) at 0.0 , $0.05,0.1,0.25,0.5,0.75,1.0,1.5$, and $2.0 \mu \mathrm{M}$. Kinetic rate constants were determined by using BIA evaluation software (version 3.0) supplied by the manufacturer.

\section{Bio-layer Interferometry}

We used bio-layer interferometry (BLI; Octet Red 384; Pall ForteBio LLC) to demonstrate the interaction of C3 with VWF enriched with ULVWF multimers (VWF/ULVWF or ULVWF). The $\mathrm{C} 3$ protein was biotinylated and captured using the High Precision Streptavidin (SAX) Biosensor, following the manufacturer's instruction. Sensors with captured C3 protein were dipped and read onto solutions containing VWF/ULVWF multimers ( $40 \mathrm{ng} / \mathrm{mL}$ ) mixed with either mouse control isotype $\operatorname{IgG}$ (25 $\mu \mathrm{g} / \mathrm{mL})$ or MR-5 (25 $\mu \mathrm{g} / \mathrm{mL})$ in PBS for 400 seconds.

\section{Results}

In - Fig. 1, the proteins used in this study are displayed by Coomassie Blue staining and western blotting using monospecific antibodies. The recombinant His-tagged VWF A1A2A3 and recombinant individual His-tagged VWF A1, $\mathrm{A} 2$, and $\mathrm{A} 3$ domains, as well as $\mathrm{C} 3, \mathrm{C} 3 \mathrm{~b}$, and $\mathrm{C} 4$, are all in highly purified form. (The rabbit anti-VWF that we used does not recognize the VWF A1 domain.)

-Fig. 2 demonstrates the results of binding studies between VWF domains and complement components, as determined using an ELISA technique with immobilized antiHis antibody. Constant concentrations of either soluble C3 or 

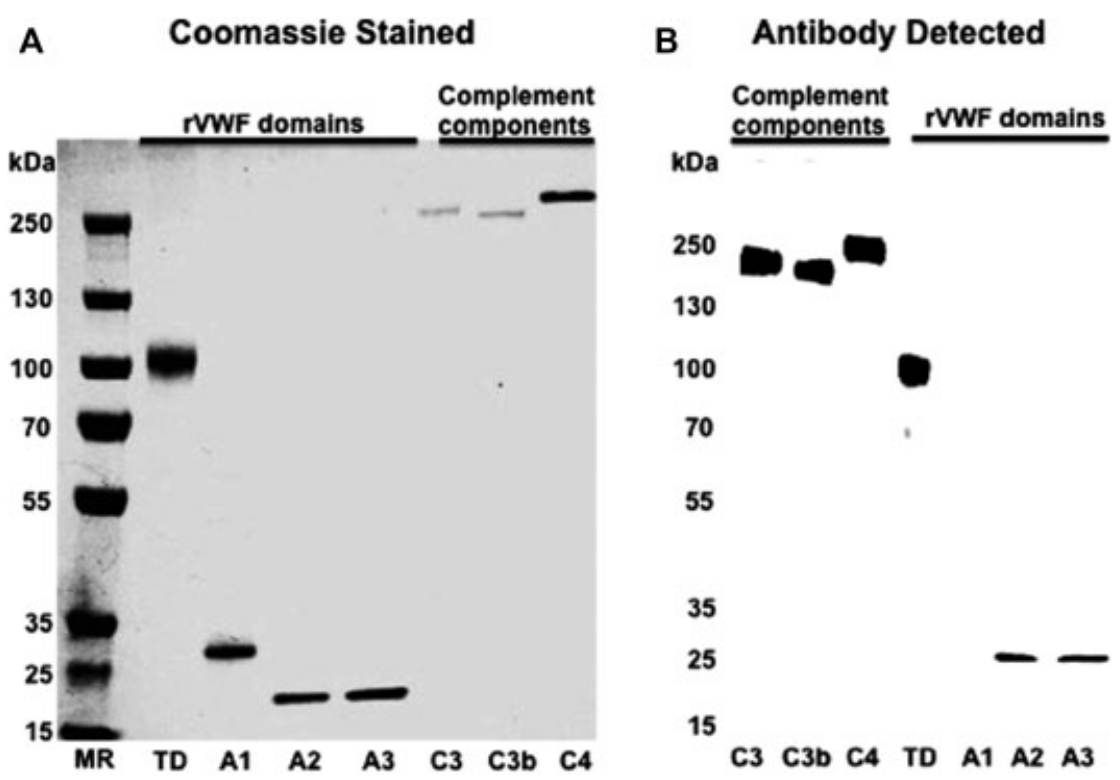

Fig. 1 Gel display of VWF and complement proteins. In (A) recombinant human VWF A1A2A3 (TD), individual VWF A1, A2, and A3 domains, and purified human C3, C3b, and C4 were electrophoresed into a 4-12\% SDS-polyacrylamide gradient gel (top left panel) and the protein bands were stained with Coomassie Blue. In (B), the same samples as in (A) were transferred onto an Immobilon-P transfer membrane (PVDF), and detected using rabbit anti-VWF plus secondary goat anti-rabbit-HRP; or with goat anti-C3 (for C3 and C3b) or goat anti-C4 plus secondary rabbit anti-goatHRP. (Note: our rabbit polyclonal anti-VWF antibody does not detect the VWF A1 domain.)

C4 $(10 \mu \mathrm{g} / \mathrm{mL})$ were bound during prolonged incubation to a range of concentrations of His-tagged VWF A1A2A3 ( -Fig. 2A). At all concentrations tested of the VWF triple domain, binding of C3 exceeded the binding of C4. Similar results were obtained when a constant concentration of soluble His-tagged VWF A1A2A3 $(100 \mu \mathrm{g} / \mathrm{mL})$ was incubated under the same conditions with a range of concentrations of C3 or C4 (-Fig. 2B).

Using the individual soluble recombinant VWFA1, A2, and A3 domains, and the same prolonged incubation before ELISA, it was determined that soluble $\mathrm{C} 3$ and $\mathrm{C} 3 \mathrm{~b}$ bind predominantly to soluble VWF A3. In contrast, soluble C4 binds predominantly to soluble VWFA1 (-Fig. 3). There was little binding of either C 3 or C4 to the VWF A2 domain.

Surface plasmon resonance experiments were conducted to determine the binding of purified $\mathrm{C} 3$ or $\mathrm{C} 4$ to recombinant VWFA1A2A3 or individual A domains ( - Table 1 and - Fig. 4). (Lower $K_{\mathrm{D}}$ values indicate higher protein-protein affinity.) The data demonstrate that: (1) C3 binds to VWFA1A2A3 with an affinity that is several-fold greater than the affinity of $\mathrm{C} 4$; (2) using individual A domains, C3 binds predominantly to A3 and C4 binds predominantly to A1; and (3) neither C 3 nor $\mathrm{C} 4$ binds to $\mathrm{A} 2$. These findings are compatible with our results obtained in the ELISA experiments.

Both the 1:1 Langmuir binding model and the shift baseline model were used to fit the kinetic data shown in - Table 1 for the binding interaction between $\mathrm{C} 3$ and $\mathrm{C} 4$ across the separate flow cell surface for VWF A1A2A3 and the individual domains, $\mathrm{A} 1, \mathrm{~A} 2$, and $\mathrm{A} 3$. Dissociation rates $\left(K_{\mathrm{D}}\right)$ and $n$ (number of experiments performed) are also shown. Kinetic analysis shows significantly higher affinity of C3 than C4 for binding to VWF A1A2A3 (-Table 1). C3 $\left(K_{\mathrm{D}}=661 \pm 290 \mathrm{nM}\right)$ exhibited a much lower $K_{\mathrm{D}}$ than $\mathrm{C} 4\left(K_{\mathrm{D}}>2 \times 10^{3} \mathrm{nM}\right)$ (-Table 1). Similarly, binding of VWF A1A2A3 to immobilized C3 $\left(K_{\mathrm{D}}\right.$ $=232 \pm 46.1 \mathrm{nM})$ showed higher affinity than binding to immobilized C4 $\left(K_{\mathrm{D}}=523 \pm 64 \mathrm{nM}\right)$ (-Table 1).

The individual A3 domain had a binding preference for $\mathrm{C} 3$ over C4, as determined by kinetic analyses. From - Table 1, the $K_{\mathrm{D}}$ for the binding of soluble $\mathrm{A} 3$ domain to immobilized C3 was $100 \pm 26 \mathrm{nM}$, and a similar $K_{\mathrm{D}}$ of $137 \pm 88 \mathrm{nM}$ was determined for the binding of soluble $\mathrm{C} 3$ to immobilized $\mathrm{A} 3$ domain. On the other hand, $\mathrm{A} 3$ had a weak binding affinity for $\mathrm{C} 4\left(K_{\mathrm{D}} \geq 2.0 \times 10^{3} \mathrm{nM}\right)$. C4 did, however, exhibited a comparable affinity for $\mathrm{A} 1\left(K_{\mathrm{D}}=139 \pm 65 \mathrm{nM}\right)$, compared with $\mathrm{C} 3\left(K_{\mathrm{D}}=252 \pm 130 \mathrm{nM}\right)$ (-Table 1). The A2 domain had little binding capacity in ELISA or no binding capacity for either C3 or C4 using SPR.

The capacity of the antibody MR-5 (monovalent Fab fragment) to block the binding of soluble VWF/ULVWF multimers (labeled "ULVWF") to C3 was assessed by utilizing BLI technology. - Fig. 5 demonstrates that MR-5 was effective in blocking the interaction between VWF/ULVWF in solution and C3 protein bound to Streptavidin biosensors. Thus, the data are compatible, even using different techniques (ELISA methods for -Figs. 2 and $\mathbf{3}$ and an advanced ELISA-like method [Octet Red 384] for - Fig. 5; surface plasmon resonance for - Figs. 4 and - Table $\mathbf{1}$ ).

\section{Discussion}

The precise event initiating AP activation has remained undefined for many years. During this time, it was 
A

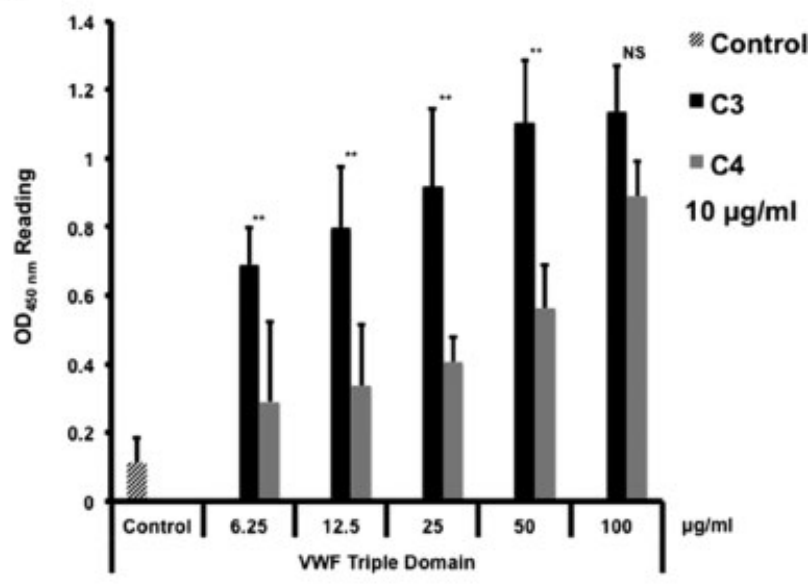

B

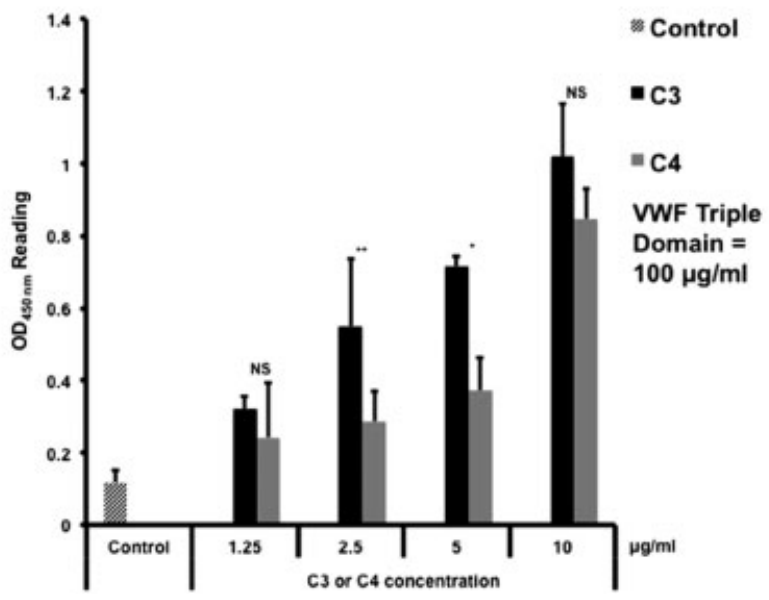

Fig. 2 Recombinant VWF-A1A2A3 triple domain binds to purified C3 and, to a lesser extent, purified $C 4$. In preliminary experiments using one immobilized protein and one soluble protein, we determined the concentration ratios that yielded maximum binding. In (A), a constant concentration of either C3 $(n=5)$ or $\mathrm{C} 4(n=5)$ was premixed with a range of VWF A1A2A3 triple domain concentrations before incubation in microwells with immobilized mouse monoclonal anti-His-antibody $(160 \mathrm{ng} / \mathrm{mL})$ to capture the His-tagged VWF A1A2A3. Subsequently, $\mathrm{C} 3$ or $\mathrm{C} 4$ bound to the VWF A1A2A3 was detected using goat antihuman C3 or goat anti-human C4 plus rabbit anti-goat HRPtagged secondary antibody and TMB. Our rabbit anti-human VWF does not recognize the VWFA1 domain (see - Fig.1); consequently, to capture with maximum effectiveness, His-tagged VWF A1A2A3 (VWF triple domain) with bound $\mathrm{C} 3$ or $\mathrm{C} 4$, we used mouse monoclonal antiHis antibody. In (B), the opposite experiments were also done. Anti-C3 or anti-C4 were immobilized on ELISA plates to capture a series of C3 or $C 4$ concentrations preincubated with a constant concentration of $100 \mu \mathrm{g} / \mathrm{mL}$ of recombinant VWF A1A2A3. The C3 or C4 bound VWF $A 1 A 2 A 3$ was detected using rabbit anti-human VWF and a secondary goat anti-rabbit/HRP. The color reaction produced by 3,3',5, '-tetramethylbenzidine was read in spectrophotometer at A450. A constant concentration of VWF A1A2A3 was premixed with a range of C3 $(n=7)$ or $C 4(n=7)$ concentrations before incubation and analysis as in (A). Controls were PBS/1\% bovine serum albumin (BSA), VWF A1A2A3, A1, A2, or A3 alone, or purified $C 3$ or $C 4$ alone at the same concentrations used in the mixes $(n=12)$. $p$-Value was calculated using Student's $t$-test. ${ }^{*} p<0.05 ;{ }^{* *} p<0.01$.

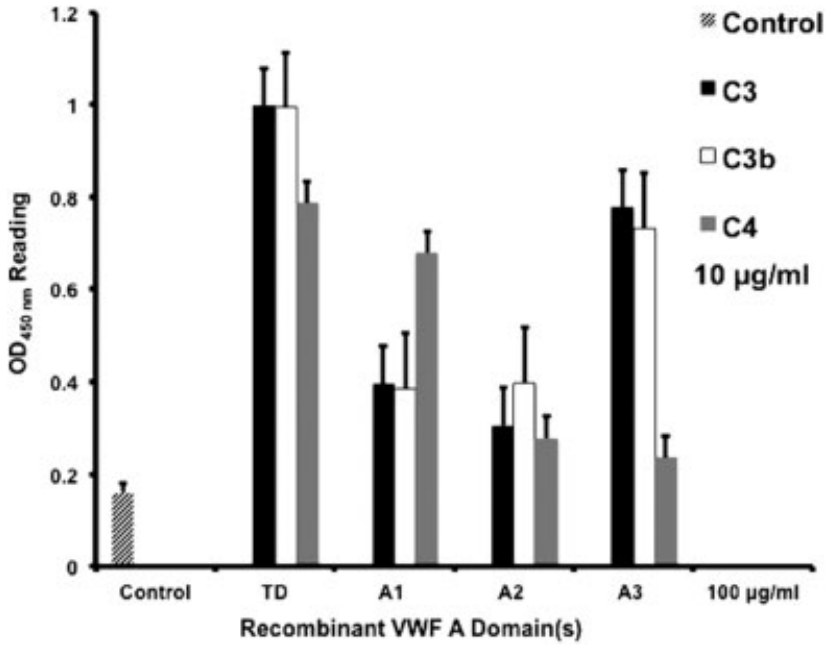

Fig. 3 Purified human C3 and C3b bind predominantly to recombinant human VWF $\mathrm{A} 3$, and purified human $\mathrm{C} 4$ binds predominantly to recombinant VWF $A 1.100 \mu \mathrm{g}$ of each VWF A domain were mixed separately with $10 \mu \mathrm{g} / \mathrm{mL}$ of either C3, C3b, or C4, and incubated at $4^{\circ} \mathrm{C}$ overnight. The mixtures were pipetted into microwells containing immobilized rabbit anti-His antibody, and incubated for 2 hours at $37^{\circ} \mathrm{C}$. The wells were washed and the quantity of $\mathrm{C} 3$ or $\mathrm{C} 4$ bound to each domain was determined using either goat anti-human $\mathrm{C} 3$ or $\mathrm{C} 4$ antibodies, as described in - Fig. $\mathbf{2}(n=7)$. Mixtures of recombinant human VWFA1A2A3 (TD) were used as positive controls. The negative control was phosphate-buffered saline (PBS)/bovine serum albumin, $\mathrm{pH}$ 7.4. $\mathrm{p}$-Value was calculated using Student's $t$-test. ${ }^{*} p<0.05 ;{ }^{* *} p<0.01$.

Table 1 Surface plasmon resonance binding

\begin{tabular}{|l|l|l|l|}
\hline $\begin{array}{l}\text { Immobilized } \\
\text { ligand }\end{array}$ & $\begin{array}{l}\text { Analyte } \\
\text { soluble }\end{array}$ & $K_{\mathrm{D}}(\mathrm{nM})$ & $\begin{array}{l}\text { Number of } \\
\text { experiments }\end{array}$ \\
\hline VWF A1A2A3 & $\mathrm{C} 3$ & $661 \pm 290$ & 4 \\
\hline VWF A-1 & $\mathrm{C} 3$ & $252 \pm 129$ & 5 \\
\hline VWF A-2 & $\mathrm{C} 3$ & No binding & 2 \\
\hline VWF A-3 & C3 & $137 \pm 88$ & 7 \\
\hline VWF A1A2A3 & C4 & $>2 \times 10^{3}$ & 4 \\
\hline VWF A-1 & C4 & $139 \pm 65$ & 8 \\
\hline VWF A-2 & C4 & No binding & 3 \\
\hline VWF A-3 & C4 & $>2 \times 10^{3}$ & 4 \\
\hline C3 & VWF A1A2A3 & $232 \pm 46.1$ & 5 \\
\hline C3 & VWF A-1 & $>2 \times 10^{3}$ & 5 \\
\cline { 2 - 4 } & VWF A-2 & No binding & 2 \\
\cline { 2 - 4 } & VWF A-3 & $100 \pm 26$ & 4 \\
\hline C4 & VWF A1A2A3 & $523 \pm 64$ & 8 \\
\hline \multirow{3}{*}{ C4 } & VWF A-1 & $504 \pm 132$ & 5 \\
\cline { 2 - 4 } & VWF A-2 & No binding & 2 \\
\cline { 2 - 4 } & VWF A-3 & $>20 \times 10^{3}$ & 4 \\
\hline
\end{tabular}

Note: The 1:1 Langmuir binding model and the shift baseline model were used to calculate the dissociation constants $\left(K_{\mathrm{D}}\right)$. 

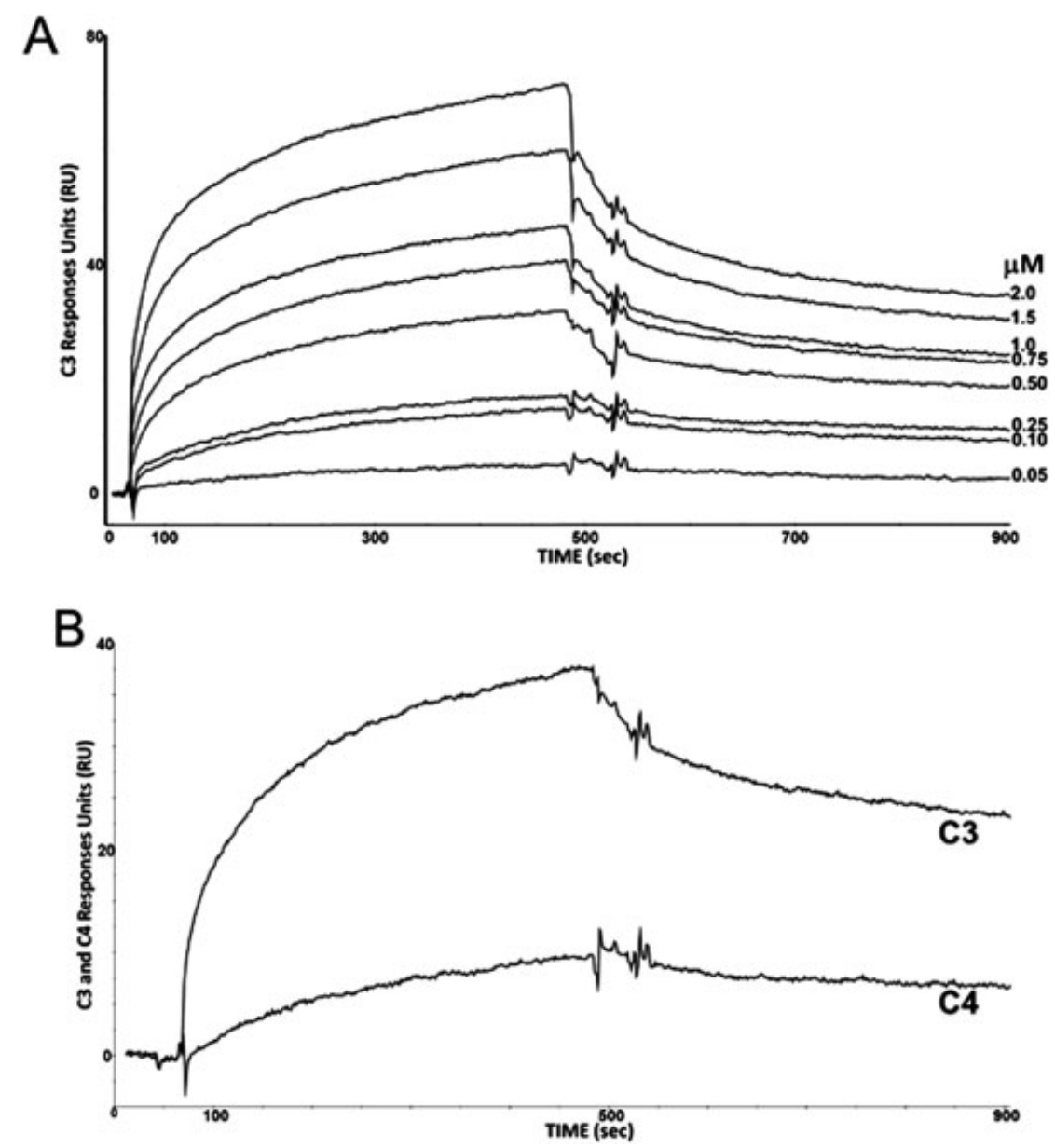

Fig. 4 Analysis of the binding of $C 3$ and $C 4$ to immobilized VWF-A3 domain using surface plasmon resonance. (A) A concentration range (as indicated) of $\mathrm{C} 3$ was analyzed across a recombinant VWF-A3 domain coupled to a biosensor surface at a flow rate of $10 \mu \mathrm{L} / \mathrm{min}$. C3 bound to VWF A3 domain with a $K_{\mathrm{D}}$ of $137 \mathrm{nM}$. (B) Sensorgrams represent the binding of C3 (500 nM) and C4 (500 nM) to immobilized recombinant VWF-A3 domain. The $\mathrm{C} 4$ had a $K_{\mathrm{D}}>2.0 \times 10^{3} \mathrm{nM}$, indicating a binding affinity for VWF A3 domain lower than C3 protein.

demonstrated $^{14-18}$ that limited activation of the AP could begin by direct hydrolysis of an intramolecular bond in $\mathrm{C} 3$. This process converted $\mathrm{C} 3$ into a hydrated form $\left[\mathrm{C} 3\left(\mathrm{H}_{2} \mathrm{O}\right)\right]$ that is capable of assuming a conformation that can cleave/

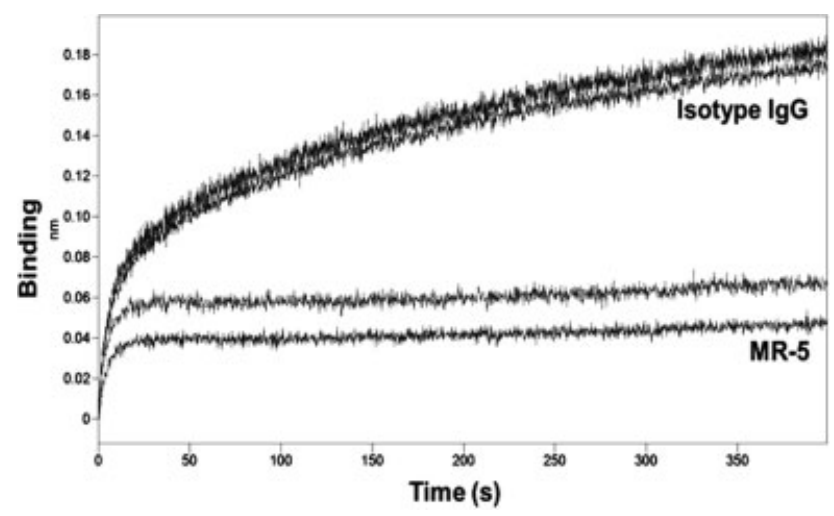

Fig. 5 Binding of VWF/ULVWF (ULVWF) to C3 protein is blocked by anti-A3 domain antibody. Biosensors coated with C3 were dipped onto wells containing a solution of ULVWF mixed with mouse $\mathrm{IgG}$ $(25 \mu \mathrm{g} / \mathrm{mL})$ or anti-VWF A3 domain antibody, MR-5 $(25 \mu \mathrm{g} / \mathrm{mL})$. The MR- 5 antibody inhibited $\sim 70 \%$ of the ULVWF-C3 binding. The graph shows two separated experiments. activate $\mathrm{C} 3$, releasing a 9-kDa fragment (C3a) to form $\mathrm{C} 3 \mathrm{~b}$. Amplification of additional C3b generation from $\mathrm{C} 3$ requires the binding of $\mathrm{C} 3 \mathrm{~b}$ [or $\mathrm{C} 3\left(\mathrm{H}_{2} \mathrm{O}\right)$ ] to an "activating cell [surface] macromolecule." 14 In previous studies, we demonstrated that EC-secreted/anchored ULVWF strings were capable of binding $\mathrm{C} 3$ (either in hydrated form or as $\mathrm{C} 3 \mathrm{~b}$ ), and initiating the assembly of the AP. In the current study, our ELISA and surface plasmon resonance experiments demonstrated that $\mathrm{C} 3, \mathrm{C} 3 \mathrm{~b}$, and, to a lesser extent, $\mathrm{C} 4$ bind to the recombinant A1A2A3 triple domain (a portion of the VWF monomer); 8,9 $\mathrm{C} 3$ and $\mathrm{C} 3 \mathrm{~b}$ were also capable of binding to the recombinant A3 domain-but not to the $\mathrm{A} 1$ or $\mathrm{A} 2$ domains.

The binding of $\mathrm{C} 3$ to VWF may depend on the conformation of the different proteins. Soluble human VWF/ULVWF multimers, recombinant human smaller VWF multimers, and recombinant human VWF dimers were used initially in pilot experiments on VWF binding to C3 or C4 (results for smaller VWF multimers and dimers are not shown). There was some binding of $\mathrm{C} 3$, and to a lesser extent, $\mathrm{C} 4$, to the smaller VWF multimers and dimers. We previously reported ${ }^{4} \mathrm{C} 3$ attachment (and AP activation) on ULVWF multimeric strings that have been secreted by-and anchored to-the surfaces of human ECs. We do not have any data to support the activation of the AP on 
soluble VWF forms that are not anchored to ECs. In the experiments in this study, the differences in conformation between (glycosylated) VWFA1A2A3 and (nonglycosylated) VWFA3 and between experimental conditions (static vs. flow) probably determine protein-protein affinity. Importantly, the $\mathrm{C} 3$ binding to A3 domain in full-length VWF was validated employing a well-characterized monovalent (Fab fragment) antibody against the A3 domain of human VWF, MR- $5,{ }^{7}$ that blocked the binding of ULVWF multimers to captured C3 protein.

Here, we show that the A3 domain of VWF contains a major binding site for $\mathrm{C} 3$ protein. This result is consistent with a recent study that reported the binding of $\mathrm{C} 3 \mathrm{~b}$ protein to immobilized recombinant $\mathrm{A} 3$ domain protein. ${ }^{19}$ With the exception of collagen types I and III $^{20-22}$ and thrombospondin, ${ }^{23,24}$ no other ligands have been described for the A3 domain. This domain does not require a conformational change to enable it to bind to exposed collagen in the subendothelium. That is, the A3 domain in ULVWF strings is already in the conformation that allows collagen binding. However, the A3 domain may exist in highand low-affinity conformations available for binding to $\mathrm{C} 3$ or $\mathrm{C} 3 \mathrm{~b}$, as described for the VWF-A-like domain in factor $\mathrm{B}$ (FB). ${ }^{25,26}$ In fact, the binding site of $\mathrm{C} 3 \mathrm{~b}$ has been identified in the FB VWF-A-like domain, which has a $15.3 \%$ sequence identity with VWF-A3 domain. ${ }^{27}$ In previous experiments demonstrating C3 binding to HUVEC-anchored ULVWF strings, ${ }^{4}$ the concentration of $\mathrm{C} 3$ released into the HUVEC supernatant under the experimental conditions used (1 mL PBS, 15-minute incubation) was below about 5 pM. ${ }^{28,29}$ The $K_{\mathrm{D}}$ of C 3 for ULVWF strings would be, therefore, of the order of pM. Our surface plasmon resonance data demonstrate that the $K_{\mathrm{D}}$ of $\mathrm{C} 3$ to $\mathrm{A} 3$ or VWF A1A2A3 is approximately 100 to $600 \mathrm{nM}$. The differences in $K_{\mathrm{D}}$ suggest that the isolated $\mathrm{A} 3$ domain, as well as the $\mathrm{A} 3$ domain as a component of $\mathrm{A} 1 \mathrm{~A} 2 \mathrm{~A} 3$, assume a different conformation than the conformation exposed in EC-anchored ULVWF multimeric strings.

The results obtained from using our recombinant A2 domain protein contrast with those recently reported by Bettoni et al. ${ }^{19}$ Their study demonstrated the binding of soluble $\mathrm{C} 3 \mathrm{~b}$ to the three A domains of VWF, and indicated that the main binding site for $\mathrm{C} 3 \mathrm{~b}$ protein is located in the $\mathrm{A} 2$ domain of VWF. In our study, the C3 protein also bound the three A domains, but it had the lowest or no binding activity for the recombinant A2 domain. A possible explanation for this discrepancy is that our A2 is 20 amino acid residues longer than the protein used by Bettoni et al. ${ }^{19}$ The additional amino acid residues may alter the structural conformation adopted by the recombinant $\mathrm{A} 2$ domain $^{30}$ when tested under different conditions (e.g., static [ELISA] vs. flow [SPR]). Another possible explanation for the discrepancy is that we utilized C3 protein, whereas Bettoni et al used C3b protein in their binding analyses. On the other hand, our isolated A2 domain protein had a weak binding activity for C3 in solution (shown in - Fig. 3), while, in sharp contrast, a protein-protein interaction between the A2 domain and C3 protein was not detected by using SPR. These outcomes suggest that the covalent coupling of either A2 or C3 protein to the chip impairs the recognition sites for the potential A2C3 binding. Further studies may clarify the results.
Both hydrated C3 (designated "C3" in this study) and C3b may bind to surfaces, including ULVWF-secreted/ECanchored strings covalently. These covalent bonds may be via an exposed thioester in C3 or C3b to hydroxyl-containing amino acids (threonine, serine, and tyrosine) onto an activating surface $^{14}$ (including anchored ULVWF strings). C3 or $\mathrm{C} 3 \mathrm{~b}$ molecules on anchored ULVWF strings then bind FB to produce $\mathrm{C} 3 \mathrm{~B}$ or $\mathrm{C} 3 \mathrm{bB} .{ }^{17,18} \mathrm{FB}$ in the $\mathrm{C} 3 \mathrm{~B}$ or $\mathrm{C} 3 \mathrm{bB}$ complex is cleaved to active $\mathrm{Bb}$ by factor $\mathrm{D}$, generating $\mathrm{C} 3 \mathrm{Bb}$ or $\mathrm{C} 3 \mathrm{bBb}$ (the active AP C3 convertases).

Attempts to design drugs targeting the exposed thioester in $\mathrm{C} 3 \mathrm{~b}$ as a means to inhibit the complement pathways have been explored by Sahu et al, ${ }^{31}$ and further studied by Sahu and Pangburn. ${ }^{32}$ The cleavage by hydrated $\mathrm{C} 3$ of $\mathrm{C} 3$ into $\mathrm{C} 3 \mathrm{~b}$ can be accelerated by the interaction with a surface. ${ }^{33}$ ULVWF strings that are secreted by, and anchored to, stimulated human ECs provide important surfaces that promote $\mathrm{C} 3$ and AP activation. ${ }^{34}$

In our previous experiments using HUVEC-anchored ULVWF multimeric strings, C4 did not bind to the strings. This indicated that neither the classical nor lectin complement pathways were activated by this mechanism. ${ }^{4}$ In addition, a recent study also showed that the $C 4$ protein did not bind to individual A1, A2, or A3 domain proteins. ${ }^{19}$ In contrast, in the current study we found that $C 4$ had weak binding for the (glycosylated) VWF A1A2A3. C4 did, however, bind to the individual, isolated (nonglycosylated) A1 domain with high affinity (but not to individual A2 or A3 domains). In ECanchored ULVWF strings, the A1 domain is glycosylated (as in our recombinant VWFA1A2A3). It is likely that glycosylation in A1 domain weakens the binding of C4 to human EC-secreted and EC-anchored ULVWF multimeric strings. By analogy, the glycosylated single A1 domain binds to its major ligand, the platelet receptor glycoprotein (GP) Ib, with a binding affinity that is less than the affinity for nonglycosylated A1 domain. ${ }^{35}$ On the other hand, the natural interaction between the A2 domain and $\mathrm{A} 1$ domain inhibits the binding of $\mathrm{A} 1$ domain to GPIb in the context of VWF A1A2A3 triple domain protein. 8,36 It is well established that immobilization of recombinant VWF A1A2A3 protein disrupts the A2-A1 interaction, exposing the cryptic site for GPIb. ${ }^{8,37}$ Thus, it is also probable that this domain-domain interaction blocks the access of $\mathrm{C} 4$ for its site within the A1 domain.

In summary, this study reports the differential binding of C3, C3b, and C4 to recombinant glycosylated VWFA1A2A3, and demonstrates that $\mathrm{C} 3$ possesses a greater binding affinity for the VWF A3 domain in comparison to C4. Similarly, glycosylated VWF A1A2A3 exhibited a much higher binding affinity for $\mathrm{C} 3$ than for $\mathrm{C} 4$. These results, together with the capacity of the anti-A3 domain monovalent antibody to block the binding of ULVWF multimers to $\mathrm{C} 3$ protein, indicate that the $\mathrm{A} 3$ domain in the A1A2A3 complex of ULVWF multimers serves as the docking site where $\mathrm{C} 3$ binds and initiates the assembly and activation of the AP. Our experiments suggest that inhibition of the initiation of the AP in inflammatory conditions might be impaired by a compound that blocks the binding of C3 to A3 domains in ULVWF multimeric strings that have been secreted by, and anchored to, human ECs. 


\section{Authors' Contributions}

J.G.N., Q.D., S.C., and L.H.N. performed experiments, collected and interpreted data, and helped in writing the manuscript; Z.M.R generated the monoclonal antibody against the $\mathrm{A} 3$ domain, and helped write the paper; J.L.M. designed experiments, analyzed data, and helped write the paper; M.A.C. contributed ideas for experiments, reagents, and helped write the paper.

\section{Conflict of Interest}

None declared.

\section{Notes}

The contents of this manuscript do not represent the views of the Department of Veterans Affairs or the United States Government.

\section{Funding}

This work was supported by grants from the Mary $R$. Gibson Foundation, the Mabel and Everett Hinkson Memorial Fund, the Alkek Foundation, NIH/NIGMS GM112806, and NIH/NINDS NS-094280.

\section{References}

1 Ruggeri ZM. Mechanisms initiating platelet thrombus formation. Thromb Haemost 1997;78(01):611-616

2 Dong JF, Moake JL, Nolasco L, et al. ADAMTS-13 rapidly cleaves newly secreted ultralarge von Willebrand factor multimers on the endothelial surface under flowing conditions. Blood 2002;100 (12):4033-4039

3 Turner NA, Nolasco L, Ruggeri ZM, Moake JL. Endothelial cell ADAMTS-13 and VWF: production, release, and VWF string cleavage. Blood 2009;114(24):5102-5111

4 Turner NA, Moake J. Assembly and activation of alternative complement components on endothelial cell-anchored ultralarge von Willebrand factor links complement and hemostasisthrombosis. PLoS One 2013;8(03):e59372

5 Chen ZA, Pellarin R, Fischer L, et al. Structure of complement C3 (H2O) revealed by quantitative cross-linking/mass spectrometry and modeling. Mol Cell Proteomics 2016;15(08):2730-2743

6 Andersson J, Ekdahl KN, Larsson R, Nilsson UR, Nilsson B. C3 adsorbed to a polymer surface can form an initiating alternative pathway convertase. J Immunol 2002;168(11):5786-5791

7 Roth GJ, Titani K, Hoyer LW, Hickey MJ. Localization of binding sites within human von Willebrand factor for monomeric type III collagen. Biochemistry 1986;25(26):8357-8361

8 Auton M, Sowa KE, Smith SM, Sedlák E, Vijayan KV, Cruz MA. Destabilization of the A1 domain in von Willebrand factor dissociates the A1A2A3 tri-domain and provokes spontaneous binding to glycoprotein Ibalpha and platelet activation under shear stress. J Biol Chem 2010;285(30):22831-22839

9 Lo NC, Turner NA, Cruz MA, Moake J. Interaction of Shiga toxin with the A-domains and multimers of von Willebrand Factor. J Biol Chem 2013;288(46):33118-33123

10 Arya M, Anvari B, Romo GM, et al. Ultralarge multimers of von Willebrand factor form spontaneous high-strength bonds with the platelet glycoprotein Ib-IX complex: studies using optical tweezers. Blood 2002;99(11):3971-3977

11 Kumar RA, Moake JL, Nolasco L, et al. Enhanced platelet adhesion and aggregation by endothelial cell-derived unusually large multimers of von Willebrand factor. Biorheology 2006;43(05):681-691

12 Cruz MA, Chen J, Whitelock JL, Morales LD, López JA. The platelet glycoprotein Ib-von Willebrand factor interaction activates the collagen receptor alpha2beta1 to bind collagen: activationdependent conformational change of the alpha2-I domain. Blood 2005;105(05):1986-1991

13 Morales LD, Martin C, Cruz MA. The interaction of von Willebrand factor-A1 domain with collagen: mutation G1324S (type 2M von Willebrand disease) impairs the conformational change in A1 domain induced by collagen. J Thromb Haemost 2006;4(02):417-425

14 Law SK, Levine RP. Interaction between the third complement protein and cell surface macromolecules. Proc Natl Acad Sci U S A 1977;74(07):2701-2705

15 Pangburn MK, Ferreira VP, Cortes C. Discrimination between host and pathogens by the complement system. Vaccine 2008;26 (Suppl 8):I15-I21

16 Law SK, Dodds AW. The internal thioester and the covalent binding properties of the complement proteins C3 and C4. Protein Sci 1997;6(02):263-274

17 Schreiber RD, Pangburn MK, Lesavre PH, Müller-Eberhard HJ. Initiation of the alternative pathway of complement: recognition of activators by bound C3b and assembly of the entire pathway from six isolated proteins. Proc Natl Acad Sci U S A 1978;75(08): 3948-3952

18 Fearon DT, Austen KF, Ruddy S. Formation of a hemolytically active cellular intermediate by the interaction between properdin factors $\mathrm{B}$ and $\mathrm{D}$ and the activated third component of complement. J Exp Med 1973;138(06):1305-1313

19 Bettoni S, Galbusera M, Gastoldi S, et al. Interaction between multimeric von Willebrand factor and complement: a fresh look to the pathophysiology of microvascular thrombosis. J Immunol 2017;199(03):1021-1040

20 Lankhof H, van Hoeij M, Schiphorst ME, et al. A3 domain is essential for interaction of von Willebrand factor with collagen type III. Thromb Haemost 1996;75(06):950-958

21 Daidone V, Saga G, Barbon G, et al. The p.R1819_C1948delinsS mutation makes von Willebrand factor ADAMTS13-resistant and reduces its collagen-binding capacity. Br J Haematol 2015;170 (04):564-573

22 Legendre P, Navarrete AM, Rayes J, et al. Mutations in the A3 domain of von Willebrand factor inducing combined qualitative and quantitative defects in the protein. Blood 2013;121(11): 2135-2143

23 Wang A, Liu F, Dong N, et al. Thrombospondin-1 and ADAMTS13 competitively bind to VWF A2 and A3 domains in vitro. Thromb Res 2010;126(04):e260-e265

24 Majerus EM, Anderson PJ, Sadler JE. Binding of ADAMTS13 to von Willebrand factor. J Biol Chem 2005;280(23):21773-21778

25 Harris CL, Abbott RJ, Smith RA, Morgan BP, Lea SM. Molecular dissection of interactions between components of the alternative pathway of complement and decay accelerating factor (CD55). J Biol Chem 2005;280(04):2569-2578

26 Forneris F, Ricklin D, Wu J, et al. Structures of C3b in complex with factors $B$ and $D$ give insight into complement convertase formation. Science 2010;330(6012):1816-1820

27 Hinshelwood J, Spencer DI, Edwards YJ, Perkins SJ. Identification of the $\mathrm{C} 3 \mathrm{~b}$ binding site in a recombinant vWF-A domain of complement factor B by surface-enhanced laser desorption-ionisation affinity mass spectrometry and homology modelling: implications for the activity of factor B. J Mol Biol 1999;294(02):587-599

28 Coulpier M, Andreev S, Lemercier C, et al. Activation of the endothelium by IL-1 alpha and glucocorticoids results in major increase of complement $\mathrm{C} 3$ and factor $\mathrm{B}$ production and generation of C3a. Clin Exp Immunol 1995;101(01):142-149

29 Hoie EB, McGuire TR, Leuschen PM, Zach TL. Pentoxifylline inhibits tumor necrosis factor-alpha induced synthesis of complement component C3 in human endothelial cells. Biol Pharm Bull 2004;27(10):1670-1673

30 Auton M, Cruz MA, Moake J. Conformational stability and domain unfolding of the Von Willebrand factor A domains. J Mol Biol 2007;366(03):986-1000 
31 Sahu A, Pangburn MK. Investigation of mechanism-based inhibitors of complement targeting the activated thioester of human C3. Biochem Pharmacol 1996;51(06):797-804

32 Sahu A, Rawal N, Pangburn MK. Inhibition of complement by covalent attachment of rosmarinic acid to activated C3b. Biochem Pharmacol 1999;57(12):1439-1446

33 Nilsson B, Nilsson Ekdahl K. The tick-over theory revisited: is C3 a contact-activated protein? Immunobiology 2012;217(11): $1106-1110$

34 Turner N, Nolasco L, Nolasco J, Sartain S, Moake J. Thrombotic microangiopathies and the linkage between von Willebrand factor and the alternative complement pathway. Semin Thromb Hemost 2014;40(05):544-550
35 Cruz MA, Handin RI, Wise RJ. The interaction of the von Willebrand factor-A1 domain with platelet glycoprotein Ib/IX. The role of glycosylation and disulfide bonding in a monomeric recombinant A1 domain protein. J Biol Chem 1993;268(28): 21238-21245

36 Aponte-Santamaría C, Huck V, Posch S, et al. Force-sensitive autoinhibition of the von Willebrand factor is mediated by interdomain interactions. Biophys J 2015;108(09):2312-2321

37 Martin C, Morales LD, Cruz MA. Purified A2 domain of von Willebrand factor binds to the active conformation of von Willebrand factor and blocks the interaction with platelet glycoprotein Ibalpha. J Thromb Haemost 2007;5(07): 1363-1370 